\title{
Hepatic Cirrhosis: Diagnostic and Prognosis in Internal Medicine
}

\author{
Demba Diédhiou*, Djiby Sow, Elhadji Mamadou Moussa Thioye, Michel Assane Ndour, \\ Ibrahima Mané Diallo, Fatou Kiné Gadji, Anna Sarr, Maimouna Ndour Mbaye \\ Department of Internal Medicine II, University Hospital Center of Dakar, Cheikh Anta Diop University, Dakar, Sénégal \\ Email: *dembadiedhiou1976@gmail.com,drdjiby@yahoo.fr, elthioye92@gmail.com, michelassanendour@yahoo.fr, \\ ibrahimamanediallo@hotmail.fr,kinegadji@gmail.com, annasarr@orange.sn,mbayester@gmail.com
}

How to cite this paper: Diédhiou, D., Sow, D., Thioye, E.M.M., Ndour, M.A., Diallo, I.M., Gadji, F.K., Sarr, A. and Mbaye, M.N. (2020) Hepatic Cirrhosis: Diagnostic and Prognosis in Internal Medicine. Open Journal of Internal Medicine, 10, 171-180. https://doi.org/10.4236/ojim.2020.102018

Received: December 25, 2019

Accepted: May 10, 2020

Published: May 13, 2020

Copyright ( 2020 by author(s) and Scientific Research Publishing Inc. This work is licensed under the Creative Commons Attribution International License (CC BY 4.0).

http://creativecommons.org/licenses/by/4.0/

(c) (i) Open Access

\begin{abstract}
Introduction: Cirrhosis is a public health problem and the causes are dominated by viral hepatitis and alcoholism. Deaths due to cirrhosis represented $2.4 \%$ of deaths worldwide in 2017 . The aim of this work was to study the diagnostic and prognosis of cirrhosis at the department of internal medicine of the Abass Ndao hospital center. Patients and Methods: This is a retrospective, descriptive study, carried out on the basis of patient files followed from May 1, 1999 to February 28, 2018 (19 years) and included all patients for whom the diagnosis of cirrhosis was accepted. The diagnosis of cirrhosis was made on the basis of clinical, biological and ultrasound (portal hypertension, signs of hepatocellular insufficiency, a diffuse heterogeneous aspect, and irregularity of the liver contours). Results: 60 patient files were listed with an average age of 46.9 years, a sex ratio of 2.2 and alcoholism in $11.7 \%$ of the cases. The reasons for consultation were dominated by an increase in the size of the abdomen (55\%), lose weight (50\%). On physical examination, it was a hepatomegaly (43.3\%) and jaundices (33.3\%). Exploration of liver function showed a cholestasis (48\%), a cytolysis (58\%) and a hepatocellular insufficiency (46.2\%). Anemia was found in (24\%). The abdominal ultrasound found in all cases a dysmorphic, heterogeneous liver with irregular contours. Hepatic atrophy was found in $20 \%$. Among the 34 patients who underwent upper gastrointestinal endoscopy, they were grade 2 to 3 esophageal varicose vein in $67.6 \%$, an erosive gastropathy in $29.4 \%$. The etiology was viral hepatitis B in $45 \%$, alcoholism in $11.7 \%$ and undetermined in $43.3 \%$. A hematemesis complicated the evolution in $16.7 \%$. Carcinomatous degeneration was found in $5 \%$ and $16.7 \%$ of the patients had died. Conclusion: our experience with cirrhosis was marked by advanced forms at the stage of complications. The viral etiology B remains dominant. Prevention will involve early detection and vaccination.
\end{abstract}




\section{Keywords}

Cirrhosis, Diagnosis, Internal Medicine, Senegal

\section{Introduction}

Cirrhosis of the liver is a disease defined as a diffuse disorganization of the liver architecture, with annular fibrosis delimiting nodules of hepatocytes in clusters, called regeneration nodules [1]. It can affect anyone with chronic liver disease. Multiple etiologies are dominated by chronic viral hepatitis B and C, alcohol-related liver disease and non-alcoholic steatohepatitis [2]. According to the Global Burden of Disease Study, there were 10.6 million cases of decompensated cirrhosis. Cirrhosis is a public health problem in many countries. Despite the availability of effective treatments for the prevention and treatment of hepatitis $\mathrm{B}$ and $\mathrm{C}$, they were still the leading causes of cirrhosis worldwide, particularly in low-income countries [3]. This destructive liver disease can be life-threatening from these various complications, such as hepatocellular failure or hepatocellular carcinoma. Cirrhosis is the leading cause of liver-related death worldwide [4]. In 2017, cirrhosis caused more than 1.32 million deaths. Deaths due to cirrhosis accounted for $2.4 \%$ of all deaths worldwide in 2017. Sub-Saharan Africa had the highest age-standardized mortality rate (32.2 deaths per 100,000 populations) [3]. In sub-Saharan Africa, particularly in Senegal, few studies have focused on the manifestations of cirrhosis in internal medicine department. The aim of this work was to study the diagnostic and evolutionary aspects of cirrhosis of the liver at the Medical Clinic II of the Abass Ndao Hospital Center.

\section{Patients and Methods}

The study was carried out at the Abass Ndao hospital center in Dakar in the hepato-gastro-enterology and internal medicine departments. It is a retrospective, descriptive study based on the files of cirrhotic patients followed up in hepato-gastro-enterology consultations and the files of patients admitted to hospitalization, in the internal medicine department, from 1st May, 1999 to February 28, 2018 (19 years). We included all patients for whom the diagnosis of cirrhosis was accepted. The diagnosis of cirrhosis was made on the basis of a cluster of arguments including: data from the clinical liver examination and the existence of portal hypertension, signs of hepatocellular insufficiency, ultrasound data (diffuse heterogeneous aspect, irregularity of the liver contours), data from the histology of the liver, when the liver biopsy was performed. Portal hypertension was defined by a syndrome composed of ascites, esophageal varices, collateral venous circulation, and splenomegaly. Hepatocellular insufficiency was based on the existence of clinical manifestations (asthenia, hepatic fetor, hepatic encephalopathy, jaundice, stellar angioma, leukonychia, palmar erythrosis, digital hippocratism, ascites, and asterixis) and biological manifestations (lowering of 
prothrombin level with negative Köhler test, hypo albuminemia, hypoglycemia, hyper biluribinemia). Progressive complications were also sought. Carcinomatous degeneration was retained in front of an alteration of the general state, a painful cirrhotic liver, hyperthrophy of lymph nodes (Troisier's lymph nodes), a rise of the alpha foeto-protein $>200 \mathrm{ng} / \mathrm{ml}$ and a nodular, irregular liver and /or invasion of the vessels on ultrasound. Hepatorenal syndrome was defined as an increase in plasma creatinine greater than $130 \mathrm{mmol} / \mathrm{l}$ or creatinine clearance < $40 \mathrm{~mL} / \mathrm{min}$ excluding diuretic administration and any other cause of impaired renal function. Anemia is defined as a hemoglobin level of less than $12.9 \mathrm{~g} / \mathrm{dl}$ in men and less than $11.9 \mathrm{~g} / \mathrm{dl}$ in women. It was microcytosis if mean corpuscular volume $(\mathrm{MCV})<80 \mathrm{fl}$, normocytosis if MCV between 82 and $100 \mathrm{fl}$ and macrocytosis above a MCV of $100 \mathrm{fl}$. We did not include cirrhotic patients with incomplete records, especially when the data supporting the diagnosis of cirrhosis were incomplete. For each patient included, a survey form was completed according to the following data:

- Socio-demographic data: Age, sex, chronic alcoholism, chronic smoking, use of herbal medicine, personal and family history (first-degree relatives tested for jaundice or known liver disease).

- Clinical data: Altered general condition, infectious syndrome, signs of cholestasis, cytolysis, hepatocellular insufficiency, portal hypertension, renal insufficiency, clinical features of liver, spleen, lymphadenopathy, data from examination of other organs;

- Paraclinical data: Blood count, transaminases, gamma glutamyl transferase, alkaline phosphatases, prothrombin level, international normalized Ration, albuminemia, total and conjugated bilirubinemia, alpha-foetoprotein, creatinine and glomerular filtration rate, blood ionogram, fasting blood glucose. Other biological parameters were requested according to the profile. The results of the ascites fluid study (cytology, bacteriology and fluid biochemistry) as well as virological data: viral hepatitis $\mathrm{B}$ antigen or antibody (HBs $\mathrm{Ag}, \mathrm{HBc} \mathrm{Ac}$, viral B DNA, HBs Ac), viral hepatitis $\mathrm{C}$ antibody (HCV Ac), viral hepatitis $\mathrm{C}$ antibody (HDV Ac) were also evaluated. Imaging included abdominal ultrasound, liver CT scan, upper GI endoscopy. Fibroscan and liver puncture biopsy with histology were also reported if performed.

Data entry was done using EXCEL. The analysis was done by IBM SPSS Statiscal 24.0 and EXCEL.

\section{Results}

\subsection{Socio-Demographic Data}

During the period of our study, 60 patient records were identified. These included 17 files from internal medicine hospitalization and 43 from hepato-gastroenterology consultation. The age range of 26 to 35 years was the most represented (26.6\%). A history of jaundice was found in 1 patient $(1.6 \%)$ and hematemesis in 3 patients (5\%). Two patients had pulmonary tuberculosis (3.3\%). Chronic carrier of 
HBs antigen was found only in 1 patient's brother (1.6\%). Table 1 shows the socio-demographic and the reasons for consultation in our population.

\subsection{Clinical Data}

\section{Physical Examination Data:}

On hepatic examination, it was hepatomegaly in 26 patients (43.3\%), an irregular liver, with a sharp lower edge in $46 \%$ of cases, hard in 6 cases (10\%), painful in 6 cases $(10 \%)$ and painless in 20 cases $(33.3 \%)$. The elements of portal hypertension syndrome were found. Among the cases of portal hypertension, ascites, at the clinical examination, were the most frequent sign found in 24 patients $(40 \%)$. It was large volume in 11 patients and moderate volume in 13 patients. Splenomegaly was present in 16 patients (26.6\% of cases). According to Hackett's classification, splenomegaly was grade 1 (6.3\%), grade 2 (37.5\%), grade 3 (50\%) and grades 4 and 5 (6.2\%). Collateral venous circulation was found in 4 patients (6.6\% of cases). An edema of the lower limbs was found in 23 patients (38.3\%). Twenty-two patients (36.6\%) had hepatocellular insufficiency syndrome. Jaundice was the most frequent sign (20 patients or $33.3 \%$ of cases).

Table 1. Distribution of patients by sociodemographic aspect and reason for hospitalization.

\begin{tabular}{|c|c|c|c|}
\hline & & Effective $(n=60)$ & Frequency \\
\hline \multirow{7}{*}{$\begin{array}{l}\text { Socio-demographic } \\
\text { aspect }\end{array}$} & Mean age & \multicolumn{2}{|c|}{46.9 years (extremes of 19 and 82 ) } \\
\hline & Patient between 18 and 45 years & 32 & $53 \%$ \\
\hline & Sex ratio $(H / F)$ & & 2.2 \\
\hline & Alcohol consumption & 7 & $11.7 \%$ \\
\hline & Smoking & 8 & $13.3 \%$ \\
\hline & High blood pressure & 6 & $10.0 \%$ \\
\hline & Diabetes mellitus & 3 & $05.0 \%$ \\
\hline \multirow{11}{*}{$\begin{array}{l}\text { Reasons for } \\
\text { hospitalization }\end{array}$} & Abdominal volume increase & 33 & $55.0 \%$ \\
\hline & Asthenia, anorexia, slimming & 30 & $50.0 \%$ \\
\hline & Jaundice & 10 & $16.6 \%$ \\
\hline & Hematemesis & 10 & $16.6 \%$ \\
\hline & Edema of the lower limbs & 10 & $16.6 \%$ \\
\hline & Abdominal pain & 6 & $10.0 \%$ \\
\hline & Reference for cirrhosis & 5 & $8.3 \%$ \\
\hline & Itching & 4 & $6.6 \%$ \\
\hline & Monitoring viral hepatitis B & 3 & $5.0 \%$ \\
\hline & Reference for esophageal varices & 1 & $1.6 \%$ \\
\hline & Vomiting & 1 & $1.6 \%$ \\
\hline
\end{tabular}




\subsection{Paraclinical Data}

Data from liver function test showed cholestasis in $48 \%$ of cases, cytolysis in $58 \%$. The biological exploration of hepatocellular insufficiency found a decrease in prothrombin level $(<70 \%)$ in $46.2 \%$ of the 26 patients it was searched for, a low albuminemia $(<35 \mathrm{~g} / \mathrm{l})$ in $72.7 \%$ of the 11 patients it was searched for. On the hemogram, there was hyperleukocytosis (13\%), leukopenia (23.9\%) and thrombocytopenia (50\%). Anaemia was found in 24\%. Abdominal ultrasound was performed on all our patients. It found in all cases a dysmorphic and heterogeneous liver with irregular contours. Hepatic atrophy was found in 12 patients. Table 2 shows the ultrasound data. Thirty-four patients had undergone upper gastrointestinal endoscopy (57\% of cases). All results were pathological. Table 3 shows the upper gastrointestinal endoscopy data.

Table 2. Patient distribution by sonographic signs of the liver.

\begin{tabular}{llcc}
\hline \multicolumn{1}{c}{ Ultrasound parameters } & Effective & Frequency \\
\hline Appearance of the liver & Atrophic & 12 & $20.0 \%$ \\
& Eutrophic & 22 & $36.7 \%$ \\
& Hypertrophic & 26 & $43.3 \%$ \\
& Heterogeneous & 60 & $100 \%$ \\
& Irregular & 59 & $98.3 \%$ \\
Ascites & Not described & 1 & $1.7 \%$ \\
& Large volume & 8 & $13.3 \%$ \\
Splenomegaly & Moderate volume & 6 & $10.0 \%$ \\
Portal vein thrombosis & Small volume & 11 & $18.3 \%$
\end{tabular}

Table 3. Patient distribution according to signs at upper gastrointestinal endoscopy.

\begin{tabular}{|c|c|c|c|}
\hline \multicolumn{2}{|c|}{ Endoscopic signs } & \multirow{2}{*}{$\begin{array}{c}\text { Effective } \\
4\end{array}$} & \multirow{2}{*}{$\begin{array}{c}\text { Frequency } \\
11.76 \%\end{array}$} \\
\hline Esophageal varies & Absent & & \\
\hline & Grade 1 & 8 & $23.53 \%$ \\
\hline & Grade 2 & 7 & $20.59 \%$ \\
\hline & Grade 3 & 14 & $41.18 \%$ \\
\hline & Rupture of varicose veins & 1 & $2.94 \%$ \\
\hline Red signs & & 9 & 26.47 \\
\hline \multicolumn{2}{|c|}{ Cardiotuberous varicose } & 4 & $11.76 \%$ \\
\hline \multicolumn{2}{|c|}{ Hypertensive gastropathy } & 4 & $11.76 \%$ \\
\hline \multicolumn{2}{|l|}{ Erosive gastritis } & 10 & $29.41 \%$ \\
\hline \multicolumn{2}{|c|}{ Erythematous gastritis } & 11 & $32.35 \%$ \\
\hline
\end{tabular}




\subsection{Etiological and Evolutionary Data}

In $45 \%$ of the patients, the B virus was found by the positivity of the HBs antigen and alcoholism was involved in $11.7 \%$ of the cases. Metabolic cirrhosis was strongly suspected in 1 patient, who had hypertension, associated with diabetes of an unknown type. In 26 patients (43.3\%) the cause of cirrhosis was unknown. However, among these patients, alcoholic cirrhosis was suspected in 7 patients, who had an ASAT/ALAT ratio greater than 2. Hematemesis complicated the progression of cirrhosis in 10 patients (16.6\%). Three patients had renal insufficiency. One patient had hepatic encephalopathy. Carcinomatous degeneration was found in 3 patients. Among the 60 patients, 10 died or 16.6\% of the patients.

\section{Discussion}

\subsection{Socio-Demographic Aspects}

In our internal medicine department, we collected 60 cases of cirrhosis over a period of 19 years. The mean age of our patients was 46.9 years, similar to the mean age of most African studies [5] [6] [7]. In Uganda, a lower mean age of 38 years was reported [8]. In Occident, the mean age of cirrhotic patients appears higher with respectively 54 years in Canada [9] and 60 years in France [10]. This difference can be explained by the difference in etiologies. In sub-Saharan Africa, the hepatitis B virus is the main cause and infection in acquired very early in life through vertical mother-child transmission. In Europe, on the other hand, alcoholic cirrhosis is predominant. Male predominance is known, probably in connection with higher exposure to etiological factors, particularly alcoholism [3]. But also because the seroconversion of the HBe antigen is lower in men than in women [11]. Societal beliefs, particularly religious beliefs, play a significant role in certain additive behaviors. The frequency of $11.7 \%$ alcoholism in our patients was lower in the series of Maigga et al. [12] in Mali where $1.8 \%$ of cases were found. However, this alcoholism is not always admitted by the patients. Hence the importance of systematically looking for signs of chronic alcoholism. The use of phytotherapy was respectively found in 3.3\% of our patients. In Nigeria, the consumption of herbs and roots was reported as a risk factor in $46 \%$ of patients with liver disease [13]. If it does not induce cirrhosis, it could be an aggravating factor in liver disease [14]. There are few data on the hepatotoxicity of medicinal plants. The contribution of medicinal plants in the genesis of liver disease remains underestimated in sub-Saharan Africa.

\subsection{Clinical Aspects}

Cirrhosis's circumstances of discovery in our study are dominated by ascites (55\%), hematemesis (16.7\%) and jaundice (16.7\%). In other words, cirrhosis is almost discovered in its decompensation phase. This is also the case in Uganda, where among the 85 patients received for decompensated cirrhosis, $95.3 \%$ had ascites, $36.5 \%$ had varicose bleeding and $62.4 \%$ had jaundice [8]. The reason for this late discovery is thought to be the trivialization of signs in the compensatory 
phase and the initial use of traditional medicine when liver disease is suspected. The frequency of lower limb edema is variously reported in Africa [6] [8].

In the context of hepatocellular insufficiency, jaundice was the most frequent sign with $33.3 \%$ of cases. For Pariente et al. [15], jaundice is a complication frequently indicative of cirrhotic disease. The high frequency of jaundice and ascites in the African series also reflects the advanced stage of the disease at the time of diagnosis.

\subsection{Paraclinical Data}

In our series, impaired liver function confirms the frequent diagnosis at the decompensation stage. Bacterial infections are common [16]. Indeed, during cirrhosis, there is an alteration of immune defense capacities, an increase in intestinal permeability and an intestinal microbial proliferation that favors the translocation of commensal bacteria from the digestive lumen to the portal system and systemic circulation [17]. The most frequent bacterial infections in the cirrhotic patient are ascites, urinary tract infections and pneumopathies. The high frequency of anemia seems too consistent compared to the results of Apica et al. [8] in Uganda. Anemia is predominantly microcytic, probably due to chronic hemorrhage and lack of intake.

Ultrasound data on the liver are consistent with the literature, however, with $36.7 \%$ liver atrophy in our series. The reliability of ultrasound in the diagnosis of cirrhosis of the liver is known. Liver biopsy seems unnecessary, even contraindicated, if the diagnosis of cirrhosis is clearly established from clinical and ultrasound data [18]. The majority of patients who underwent upper gastrointestinal endoscopy had esophageal varices (88.2\%), including grade $3(41.2 \%)$ and a cardiotuberous site $(11.76 \%)$. This prevalence of esophageal varices in cirrhotic is close to that found in Ghana with $90.6 \%$, of which $82.2 \%$ are grade 3 [7].

\subsection{Evolutionary Aspects}

Hematemesis by rupture of esophageal varices was the most common complication. They account for $70 \%$ of digestive hemorrhages during cirrhosis. This is why upper gastrointestinal endoscopy should be systematic in all cirrhotic patients. In the two years following the diagnosis of cirrhosis, the risk of rupture of the varicose veins is $30 \%$ in the case of medium to large varicose veins. Impaired liver function and the presence of ascites are also predictive of esophageal varicose vein rupture. Also, according to Laplace's law [19], the tension of the varicose wall is directly related to the diameter of the varicose vein and inversely proportional to the thickness of the wall. Thus, an increase in the size or a decrease in the thickness of the wall will increase the tension exerted by the varicose wall and thus the risk of bleeding.

The appearance of ascites marks a decisive turning point in the evolution of cirrhosis. Its prevalence is very high in all African series due to late diagnosis [8]. Complications of ascites can also be life-threatening. Infection of ascites fluid, 
found in $27 \%$ of the series of Dia et al. [5], is an important factor in hepatic encephalopathy.

Cirrhosis was reported in $80-90 \%$ of patients with hepatocellular carcinoma [20]. It should be systematically investigated during the surveillance of the cirrhotic patient, by ultrasound monitoring and alpha-foeto-protein assay. Unfortunately, in Africa, cirrhosis is often discovered at a late stage, when the general state of the liver is altered, with hematic ascites and a painful and nodular liver. The progression to hepatocellular carcinoma is thought to be weaker in alcoholic cirrhosis than in viral cirrhosis [21].

\subsection{Etiological Aspects}

As in our series, the hepatitis B virus is the most common cause in West Africa. The death rates caused by hepatitis B virus were $50.4 \%$ in men and $45.7 \%$ in women [3]. Compared to the rest of the world, the hepatitis B virus is found in $31.5 \%$ of men and $24 \%$ of women [3]. In the majority of the cases in our study, the viral work-up consisted only of the search for chronic HBs antigen carrier. Other markers of infection were often out of reach for most of our patients. The worldwide distribution of chronic hepatitis B and C infection justifies the predominance of viral hepatitis as a cause of cirrhosis in African countries [3].

In the West, alcohol and the hepatitis C virus are the main etiologies [3] [22]. The lack of sufficient data did not allow us to search for non-alcoholic steatosis of the liver (NASH). In West Africa, it is thought to be the etiology in $5.5 \%$ of men and $7.7 \%$ of women with cirrhosis [3]. In nearly half of the cases in our study population, no cause has been found, which may be due in part to insufficient exploration, especially for autoimmune causes.

\section{Conclusion}

We report a series of hepatic cirrhosis strongly marked by advanced forms at the stage of complications. The delay in treatment is partly explained by an initial recourse to traditional medicine. The viral etiology B remains dominant, followed by alcoholism. Monitoring is marked by many people lost to follow-up. Prevention will involve early detection and vaccination.

\section{Limitation of the Study}

Our study has certain limitations due to its retrospective nature: the absence of certain data in the files, the impossibility of making a prognostic classification, the difficulty of assessing the evolution of the data, and the difficulty of assessing the evolution of the data in the files.

\section{Conflicts of Interest}

The authors declare no conflicts of interest regarding the publication of this paper. 


\section{References}

[1] Anthony, P.P., Ishak, K.G., Nayak, N.C., Poulsen, H.E., Scheuer, P.J. and Sobin, L.H. (1978) The Morphology of Cirrhosis. Recommendations on Definition, Nomenclature, and Classification by a Working Group Sponsored by the World Health Organization. Journal of Clinical Pathology, 31, 395-414. https://doi.org/10.1136/jcp.31.5.395

[2] Ge, P.S. and Runyon, B.A. (2016) Treatment of Patients with Cirrhosis. The New England Journal of Medicine, 375, 767-777. https://doi.org/10.1056/NEJMra1504367

[3] Sepanlou, S.G., Safiri, S., Bisignano, C., Ikuta, K.S., Merat, S., Saberifiroozi, M. and Abdoli, A. (2020) The Global, Regional, and National Burden of Cirrhosis by Cause in 195 Countries and Territories, 1990-2017: A Systematic Analysis for the Global Burden of Disease Study 2017. The Lancet Gastroenterology \& Hepatology, 5, 245-266. https://doi.org/10.1016/S2468-1253(19)30349-8

[4] Roth, G.A., Abate, D., Abate, K.H., et al. (2018) Global, Regional, and National Age-Sex-Specific Mortality for 282 Causes of Death in 195 Countries and Territories, 1980-2017: A Systematic Analysis for the Global Burden of Disease Study 2017. The Lancet, 392, 1736-1788. https://doi.org/10.1016/S0140-6736(18)32203-7

[5] Dia, D., Serme, Y.K., Bassène, M.L., Halim, A., Diallo, S., Thioubou, M.A., Mbengue, M. and Diouf, M.L. (2014) Spontaneous Bacterial Peritonitis in Dakar, Senegal: Study of 55 Patients with Cirrhosis. Medecine et Sante Tropicales, 24, 55-57. https://doi.org/10.1684/mst.2013.0260

[6] Diarra, M., Konaté, A., Soukho, A., Dicko, M., Kallé, A., Doumbia, K., et al. (2010) Evolutionary Aspects of Cirrhotic Disease in a Hepatogastroenterology Department in Mali. Le Mali Médical, 1, 42-46.

[7] Duah, A., Nkrumah, K.N. and Tachi, K. (2018) Oesophageal Varices in Patients with Liver Cirrhosis Attending a Major Tertiary Hospital in Ghana. The Pan African Medical Journal, 31, 230. https://doi.org/10.11604/pamj.2018.31.230.16657

[8] Apica, B., Ocama, P., Seremba, E., Opio, K. and Kagimu, M. (2013) Decompensated Cirrhosis-Related Admissions in a Large Urban Hospital in Uganda: Prevalence, Clinical and Laboratory Features and Implications for Planning Patient Management. African Health Sciences, 13, 927-932. https://doi.org/10.4314/ahs.v13i4.10

[9] Montano-Loza, A.J., Meza-Junco, J., Prado, C.M., Lieffers, J.R., Baracos, V.E., Bain, V.G., et al. (2012) Muscle Wasting Is Associated with Mortality in Patients with Cirrhosis. Clinical Gastroenterology and Hepatology, 10, 166-173. https://doi.org/10.1016/j.cgh.2011.08.028

[10] Condat, B., Remy, A.J., Jouannaud, V., Lahmek, P., Rosa, I., Cadranel, J.F., et al. (2012) The Use of Cirrhosis Care in the Hepato-Gastroenterology Departments of French General Hospitals in 2012. Bulletin Épidémiologique Hebdomadaire, 24-25, 450-456.

[11] Allan, R., Thoirs, K. and Phillips, M. (2010) Accuracy of Ultrasound to Identify Chronic Liver Disease. World Journal of Gastroenterology, 16, 3510-3520. https://doi.org/10.3748/wjg.v16.i28.3510

[12] Maiga, M.Y., Dembele, M., Diallo, F., Traore, H.A., Traore, A.K. and Guindo, A. (2002) Upper Digestive Endoscopy in the Diagnosis of Cirrhosis. Acta Endoscopica, 32, 211-218. https://doi.org/10.1007/BF03016657

[13] Nwokediuko, S.C., Osuala, P.C., Uduma, U.V., Alaneme, A.K., Onwuka, C.C. and Mesigo, C. (2013) Pattern of Liver Disease Admissions in a Nigerian Tertiary Hospital. Nigerian Journal of Clinical Practice, 16, 339-342. 
https://doi.org/10.4103/1119-3077.113458

[14] Chalasani, N.P., Hayashi, P.H., Bonkovsky, H.L., Navarro, V.J., Lee, W.M. and Fontana, R.J. (2014) ACG Clinical Guideline: The Diagnosis and Management of Idiosyncratic Drug-Induced Liver Injury. American Journal of Gastroenterology, 109, 950-966. https://doi.org/10.1038/ajg.2014.131

[15] Chalasani, N. and Pariente, A. (2006) Ascitic Decompensation. Gastroentérologie Clinique et Biologique, 30, 870-874. https://doi.org/10.1016/S0399-8320(06)73334-6

[16] Garcia-Tsao, G. (2005) Bacterial Infections in Cirrhosis: Treatment and Prophylaxis. Journal of Hepatology, 42, S85-S92. https://doi.org/10.1016/j.jhep.2004.12.006

[17] Wiest, R. and Garcia-Tsao, G. (2005) Bacterial Translocation (BT) in Cirrhosis. Hepatology, 41, 422-433. https://doi.org/10.1002/hep.20632

[18] Wiegand, J. and Berg, T. (2013) The Etiology, Diagnosis and Prevention of Liver Cirrhosis. Deutsches Ärzteblatt International, 110, 85-91. https://doi.org/10.3238/arztebl.2013.0085

[19] Pagliaro, L., D’Amico, G., Pasta, L., Malizia, G., Tinè, F., Traina, M., et al. (1994) Portal Hypertension in Cirrhosis: Natural History. In: Bosch, J. and Groszmann, R., Eds., Portal Hypertension: Pathophysiology and Treatment, Backwell Scientific, Cambridge, 72-92.

[20] Tarao, K., Nozaki, A., Ikeda, T., Sato, A., Komatsu, H., Komatsu, T. and Tanaka, K. (2019) Real Impact of Liver Cirrhosis on the Development of Hepatocellular Carcinoma in Various Liver Diseases-Meta-Analytic Assessments. Cancer Medicine, 8, 1054-1065. https://doi.org/10.1002/cam4.1998

[21] Moosa, S.V. and Müllhauptb, B. (2015) Hepatic Cirrhosis: Potential Progression and Regression. Swiss Medical Forum, 15, 100-105.

[22] Leon, D.A. and McCambridge, J. (2006) Liver Cirrhosis Mortality Rates in Britain from 1950 to 2002: An Analysis of Routine Data. The Lancet, 367, 650.

https://doi.org/10.1016/S0140-6736(06)67924-5 